\title{
THE STAGES OF KNOWLEDGE.
}

\author{
BY PROF. ALFRED H. LLOYD.
}

\section{University of Michigan.}

Knowledge, we are told, is a growth. In conscious experience is to be seen a process that is resolvable into a number of stages. In fact in no science has the evolutional idea taken a stronger hold than in psychology.

The stages of knowledge, as commonly understood, are four, if I may reduce them somewhat. Thus: sensation, perception, conception and intuition. But an evolutional series of this kind, however well it may explain its special phenomena, is really in need of being explained itself. Mayhap it is in its entirety, in its earlier as well as in its later parts, a result of the very evolution that it would explain, and if so, the science depending upon it can hardly be too quick or too thorough in recognizing the fact. Mayhap, I say, but in truth I think the supposition is a fair report of reality, and $I$ think also that the science of psychology to-day needs to be brought to a clearer consciousness of itself in this particular respect. Hence the simple suggestions that follow in this paper.' I would reflect in a perfectly general way upon the evolutional series in the growth of knowledge, and expose it, and in the end indicate what seems to me the meaning of the change in psychology that the expose effects.

Psychologists have said of sensation, as the first stage of knowledge, that it is of the material or the physical, of the visible and the tangible and the audible and the like, being a consciousness of what the self is supposed distinctly not to be. They have said that it has for its content only the here and the now and the this, or, in other words, some particular thing in some particular place at some particular time. But the purely passive experience required by this idea of sensation, whereby, to use an old-time distinction, sensation has contained no 164 
thought, no universalizing or generalizing tendency, would have to be in unconsciousness; it would be, after all is said, only a hypothetical stage of conscious experience; as soon as it were realized, the account of it would cease to be adequate. So true is this that more recently we have found sensation commonly referred to, not as consciousness or an element of consciousness at all, but merely as an antecedent of actual consciousness, that is, either the external stimulus or the internal possibility. Sensation has come to play the part of a sort of zero of knowledge, a lower 'limit' in the evolutional scale.

Very simple experiments have demonstrated the impossibility of conscious ' sensuous' experience without the quality or meaning of the object being determined by relations beyond its position in space and beyond the moment of its being experienced and beyond individuality or isolation in general. In consciousness, however simple, say of mere color or of pressure or of temperature, other things and other times and other places determine the character of this thing here and now ; any simplest object as it enters consciousness gets outwardly reaching relations. The later idea of sensation, then, only marks a retreat in psychological doctrine before this now undisputed law of relativity. But, alas! even science can make the mistake of jumping from the frying pan into the fire, since sensation as outer stimulus or as inner possibility is, if possible, more objectionable than sensation as physical atom or element. Knowledge at zero brings fatal difficulties.

Thus the idea of sensation as stimulus only shows an attempt, very common and doubtless very natural in human thought, to keep the same relations or conditions in unconsciousness that are observed in consciousness. Sensation as stimulus carries the dualism of mind and matter or subject and object into the sphere of life that lies wholly beneath or back of conscious experience. But doing this it robs dualism of all real meaning or content. It makes dualism absolutely formal, a mere hypothesis based upon a questionable analogy. From the point of view of what is without to the conscious self, that is, from the point of view of the object, the psychologist who entertains the notion of an wholly independent stimulus to consciousness 
undertakes to present a stage or a state of life in which the distinction between what is without and what is within is quite impossible. That distinction is a development, not an antecedent condition.

The justice of this criticism is evident also from the complimentary idea of sensation as the subjective basis of possibility, that is, as a 'sensation continuum' or an originally wholly undifferentiated consciousness, a sensuous consciousness of no distinctions, whether in its object or in the organism, out of which a highly differentiated experience with organism to correspond is evolved. But surely one does not need glasses to see that 'sensation continuum ' not only is but another name for unconsciousness, but also, like sensation as stimulus, is a sort of indirect or wouldbe dualistic account of a condition in which mind and matter or subject and object are really not two but one. At least the only object to which it can claim any right is as thin a ghost as ever crossed the path of science. Sensation, then, whether as objectively a mere stimulus of consciousness or as subjectively a continuous or undifferentiated consciousness, in so far as to be regarded the first stage of knowledge, bears witness to an original state of unity or identity between self and not-self.

It sums up the foregoing to say that 'sensation continuum' and sensation as physical stimulus are, in the first place, opposites or extremes that meet, since the dualism on which their opposition depends cannot stand, and, in the second place, purely formal ideas, descriptive of the beginning of mental life only analogically and retrospectively. If you must recognize them call them limits, since, as already suggested, they show knowledge at zero; call them abstract limits, but remember that the idea of a limit always gives reality rather to a law operating within the observed members of a series than to a separate thing or a separate state. A limit never is; only the series and its law are; the limit, so to speak, only sets the law and so deepens the reality of each member of the series by making the series itself an organized whole. In mathematics the infinite and the infinitesimal are not real as quantities; they are real only as relationship within quantity or among quantities; they are quantitative indirections for quality and law. So, again, in 
psychology, sensation, at best only the infinitesimal of knowledge, whether as continuum or as stimulus, can not be real as a separate antecedent stage of knowledge; it must be, on the contrary, the epistemologist's indirection, or apology, for something deeper than mere knowledge and its stages, say for the vital principle itself or for the impulse to self-expression. The epistemologist has been ready enough to be an evolutionist, but he has also insisted on abstracting the knowing or merely cognitive self. Hence his notion of sensation as a first stage, when in reality it is not that. Psychology, in short, is more than epistemology ; it is biology also ; and sensation, as evolutional epistemology has reported it, is, I repeat, an indirection or abstraction for the principle of vital spontaneity.

Just what this principle is, in what terms it must define itself to the new psychology, the foregoing has all but indicated. Thus the law or principle to which the limits, sensation as mere stimulus and sensation continuum, testify or give reality, can have, I think, no better elementary statement than this, namely, that external stimulus and internal motive are one and identical ${ }^{1}$ and in this identity, which makes knowledge from the start, not a mere consciousness of an outer world, but at once a part of and a means to active self-expression, in brief a something on which depends the freedom of the vital impulse, in this identity lies the first law of knowledge, a law which I would have psychology substitute for its still lingering doctrine of sensation as the first stage and the infinitesimal of knowledge. As a law of knowledge it is, of course, a key to the understanding of positive or conscious sensation, which is obviously quite distinct from sensation as mere stimulus, and of perception and conception and intuition, and to the understanding also of the order that psychology has come to give to them in the growth of knowledge.

Positive or conscious sensation, to which I now turn, involves the reference of some so-called sensuous quality to some external object. In different senses the degree of this objective refer-

1 Certainly, quite apart from what has been said here, one must indeed have difficulty in entertaining the idea of a stimulus so external as not to be also motive or of a motive so internal as not to be also stimulus. 
ence varies. For example, it is commonly much greater in the experiences of the eye than in those of the organs of smell or taste, but differences in degree in no wise change the fact or principle, which is our present concern. Conscious sensation is never purely subjective. Psychologists are now well agreed on this point. The experiments alone, already referred to, which have demonstrated the absolute dependence of any sensation for its meaning or quality upon other experiences beyond its own time and place, have left them in no doubt. Some have even imagined that the relationship between different mental states could be mathematically determined; others refuse to go so far; but all accept the general law of relativity. Thus to give Höffding's very conservative wording of the law: "From the moment of its first coming into being the existence and properties of a sensation are determined by its relation to other sensations." That this is a law of the objectivity as well as of the relativity of all conscious sensation hardly needs to be indicated, but, to be perfectly explicit, I may add that 'relation to other sensations' must mean to other sensations in space as well as in time or that dependence of any particular experience for its own special meaning on other moments is also necessarily dependence on other places or positions. If the time relations tend to keep the meaning of the experience subjective, the space relations must make it objective. In other words, the simple law of relativity must be taken as signifying that all conscious sensation is of a spatially external object, or not-self, but of an object whose qualities are in a certain interesting way subjective, in that they must embody by implication, if not directly, the self's past.

But this is not final ; for, while the sensuously qualified object, or not-self, must, in the light of the law of relativity, be thought as the past self objectively present to the self, or as the self's objectified past, yet it is clear that the very fact of presentation or objectification points to some change in that past, say to some operation therein performed upon it, and an understanding of exactly what this operation is, is all-important. To get such understanding, however, we must, if possible, get back of the law of relativity or objectivity; we must get back of this law, at least so 
far as our comprehension of it depends on an application of its principle merely to differences of position in space and time or even of stimuli of measurable quantity or intensity. Relationship, let us reflect, is as much a matter of unity as of difference, of continuity as of isolation, of organic movement as of external ratios, and the objective world must in general owe its reality to something even deeper than 'difference thresholds' or 'threshold values' or than mere quantities of any kind.

The real difficulty is that so far we have been looking rather to what the sensuous object is than to how it is. The law of relativity, as sketched above, binds all experiences into one differentiated whole, and with space and time as the distinct but inseparable bases of the relations a past self as that which qualifies and a not-self as that which is qualified get their definite places in a theory of knowledge, and are seen of course to have most intimate connection each with the other; but assuredly more must be known than the formal bases of the relations. We must get at the living reality, at the vital principle of relationship instead of its formal bases. The theory of knowledge has been tarrying too long at the sign of the formal bases.

And what is the living reality, the vital principle? Plainly it is the impulse to self-expression with its identical aspect of motive and stimulus. Impulse to self-expression, at once differentiating and organizing, since both a wholly random act, an act in general, and a wholly specific act, an act in isolation, are not only unknown but also unthinkable, is a principle that quite transcends the special forms, space and time, ${ }^{1}$ and yet that, so far as manifesting itself in motion, is spatially and temporally interpretable. Action, identical with self-expression being neither random nor specific, neither general nor individual, must be as expressive of control as of spontaneity. Accompanying all action, then, there must be a tension, or, the same thing, a consciousness, consciousness being always a tension between control and impulse or spontaneity. Control, however, is just that aspect of self-expression which gives reality to a not-self

1 One has here to think of Spinoza's self-caused and self-intelligible substance, existing in se and per se, that transcended thought, the time aspect of law, and extension, the space aspect of law, in that it had infinite other attributes. Spinoza, be it remembered, all but gave Leibnitz his self-acting monad. 
with stimulating qualities, while spontaneity, as a positive impulse in tension with control, shows these qualities to be real only as embodying the past, since such qualities must be reminiscent. In short, the not-self, as qualified, does but show the self's past in tension, and whether we approach the matter from the standpoint of self-control or from that of the not-self in whose qualities the self's past is seen to be in tension, the presence of a process of organization, of an organic activity, is beyond question,' and the organization plainly is not less of the objective qualities than of the controlled activities or impulses belonging to them. But organization of any particular impulse signifies reduction of it to a pure means wholly adapted to the single end of the organism, or to such a condition that when expressed the whole self can identify itself with it ; and organization of all the manifold impulses must signify the development of activities every one of which can and must fully express the self. And on the side of the objective qualities, often called the outer stimuli, a perfectly correlative process, as already said, must take place, in that each individual quality, or sensuous content, corresponding to an individual impulse, must in its function of stimulus appeal to the whole self, not to the impulse as an isolated activity; and must therefore in its qualitative character be determined by a relation to the other qualities. Each qualitative part, or content, of the not-self, in so far as stimulus, must be at the same time, by 'fusion,' if you will, or 'assimilation' or 'association,' the qualitative whole, and just herein we see from the standpoint of impulse to self-expression, which is in action a process at once of differentiation and of organization; just herein do we see what the law of relativity means, how it is a law of objectivity, that is, a principle of control, and in being this is also a law of organic activity, and how, secondly, from the same standpoint, stimulus just in so far as it produces ' reaction' must be absolutely identical with motive.

2 Compare with this account of the origin of the not-self the extremely interesting special case of it in the generally accepted explanation of the idea of space as rising with organization and symbolization (through association of muscular and tactual and retinal sensations) of the motor-impuIses. Space as geometricalthat is, 26 mathematically definable-is the objective correlate of free movement. An exact geometry is possible, 'innate,' only to such as have the power of free movement. 
The identity of motive and stimulus, above suggested as the first law of knowledge, very materially modifies a current idea of 'reaction,' as but just now hinted, and gives a notion of environment, heretofore styled the not-self, that has far-reaching consequences alike in psychological and in ethical theory. To add a few words upon the first point, that of the proper conception of reaction, it is evident that identifying stimulus and motive reduces reaction wholly to a process of the self acting upon itself or within itself, or even of environment acting upon itself or within itself, and not to what has been so often assumed, a process of self or mind, as something essentially distinct in nature, acting or reacting in its own peculiar way upon not-self or matter. Indeed, 'reaction' is a term imbued altogether too much. with the spirit of dualism to be at all safe in self-controlled discourse. 'Self-activity' is far better, and with the conception of environment here required, whether one means self-activity of environment or self-activity of self is of no importance. "Environment did it" equals "self did it" in all cases of action, since the stimulating quality by reason of its determining relations is environment as a whole and the organically qualified environment as a stimulating whole is one and the same with the organic self and its impulse to complete self-expression. ${ }^{1}$

The notion of environment that our law of knowledge enforces is this: Environment is the self present to itself, in an other-than-it form, the otherness always signifying a tension between control and impulse to act. So much was really implied in what was said in a former paragraph, namely, that the notself must be regarded as the past self presented to the self, or the self's objectified past, or with regard to its stimulating qualities as the past in tension; but now we have clearly before us the operation performed upon that past, as implied in the very fact of the presentation or the objectification, this operation being nothing more nor less than organization into the present or ad-

'Quite another way, and to me a very suggestive way, of putting the foregoing is that the human body is to be looked upon only as a part of 'environment.' The tendency to cherish it, whether in the passing psychology of reaction or in religion-of course the two are related-must give a false idea of activity. In reality the human body is but a part of a physical whole; it is, however, a part whose activity is that of the whole; hence it has mind or soul. 
justment to the present, so that environment or not-self proves to be the past made present, its qualification being always a process of adjustment to the present. In other words, environment as differentially qualified and self as organically free and active develop together, not, as some have seemed to think, does environment with stimulating quality exist before a living self with interest in it, nor, as others have maintained, does the living self or soul antedate its environment. God did not create the world nor is man in any sense so creative, nor on the other hand is man in the ordinary understanding of the doctrine evolved out of the physical. Both the physical with its qualities, primary and secondary, and the freedom of self expression are evolved together. Evolution of course has been disposed to put the qualified world first in time, and creationism to put the fully developed spirit first in time, and which has committed the grossest anachronism it is really hard to say, since to reiterate, neither came first, or rather both were first and have kept the contemporaneity from the beginning. Thus, to suggest large-written illustrations, I can imagine man, when first assuming the erect position congratulating himself on having relegated so much of his past as was in the going on all fours to a mere object or symbol in his consciousness, and I can even feel the interest he must have taken in the new qualities and the more organic character that his world came to have for him with the change. The wanderer returning after long years to the scenes of childhood and seeing as object with emotional qualities that in which he had once lived, with which he had once wholly identified himself, could sympathize too. But how absurd it would be for either the returned wanderer or the erect man to say with materialistic evolution that out of that object as so qualified he had been evolved, or with orthodox creationism that in the object there was evidence of a fall from an ideal state to which, however, he has now at last returned. And yet upon such abstractions, upon such anachronisms, even recent psychological doctrine, in its idea of reaction and in its idea of the stimulating medium very largely relies. True, the past is in the object, or the environment, or the stimulating medium, but because the object is, and is at once organic and relationally differentiated, the 
present is there too, and if the present also the future. The object is not reminiscent merely; in being object it belongs to the present; it is, again, in so far as qualified and in so far as constructed or ordered literally ' up to date ;' it is a stimulus that is also motive; it is a revelation as well as a reminiscence, the future as well as the past.

In environment, or object, as now present to our thinking, we have of course the 'perceived world.' The study of positive or conscious sensation has brought us to a comprehension of what psychology knows as perception, the second stage of knowledge. The law of relativity, as also a law of objectivity and of organic self-expression, under which a sensuous consciousness develops into a consciousness of an ordered outer world present to a self-controlled self, under which the world of experience becomes in the technical sense a 'perceived' world, carries with it, as we have found, three things: (I) the persistence of the past or of past experience in any present consciousness, (2) a differential, or negative, qualification of consciousness, by which the objective reference springs up, and (3) an organic activity, whereby consciousness, becoming objective, gets what is commonly called symbolic character, being symbolic of the activity itself. Perception, then, is a process by which the past may be said to move over into the object and to abide there as an important phase of the present, and the percept, the self so present to itself, is the original unity of the self as an organism differentiated and in the differentiation projected as not-self. The percept, accordingly, is not-self, but very much as the band of prismatic colors is the not-self of unresolved light, or as tools and shop and materials are the not-self of the tool-using mechanic, or finally as a social community, in which labor is divided, is not-self to each one of its members. The percept is not-self, but also the incarnate self. What else can its tendency to symbolic character signify? Now perception, as the perceived image evolves into mere symbol, becomes conception and intuition. This is technical and abstract. But the meaning of images becoming mere symbols is not far to seek.

Any image in which by virtue of its being objective and 
of its sensuous qualities being organized, the past is fully adjusted to the present is become a symbol; it is a mere symbol. With the perfect adjustment the image's or object's qualities lose their reminiscent and individually stimulating character; the ' associated' past, on which the consciousness depends, comes to be so real in the present as no longer to be suggestive of the past; whereupon the reminiscent qualities lose value, except that of the relation embodied in them, and the object as a mere system of relations, an organically relational whole, becomes not an object of sensuous consciousness, but a symbol, that is, a basis of activity, and has the same relation to the life of the self in general that language with its 'parts of speech' has to the expression of thought. In an image or object or symbol so developed, the self is set free. No symbol is mere symbol that is not proved so by some action in use of it, and the action of course fulfills motive and stimulus as one.

The term language, so says this psychology, must be extended to include the object of consciousness in this sense of the used symbol. Indeed one has to think of parts of fluent activity in general instead of merely of parts of speech. In parts of fluent activity psychology sees the survival of the sensuously stimulating qualities or elements which in the evolution of experience gradually pass into mere terms in a system of relations. Indeed, in the narrow sense, what is language, if not a complex of 'dying metaphors' or ' material associations,' or ' passing reminiscences', dying or associated or passing in the interest of organization or adjustment or fluency? Yes, the whole world of perception, as it becomes symbolic, as in it the past is adjusted to the present, is essentially linguistic, the basis of fluency in action; it is language that the perceiving self can use, with this limitation that when the self uses it as language, when the self acts fluently in it instead of simply observing it, it is more properly called the world of conception, since the self is then rather conceiving than perceiving reality.

It is common enough in psychology to connect intimately conception and the use of language, but observe that here, as the term language is made to include so much more than it usually covers, there is demand also for a more inclusive idea of 
conceptual thought. All sensuous images becoming symbols are linguistic; all fluent expression of self, all free activity, is thought in the stage of conception. Language obviously is only another name for the not-self as the self incarnate. ${ }^{1}$

But to some I shall doubtless seem to be denying the very most essential function of language, which is to name or report or describe. Language, I am reminded, enables its user to stand aloof from the physical world and to carry on an abstract activity - with reference to the world, it is true, but quite apart from it. So separate from ordinary activity has language been regarded that it has even been declared to be a gift of heaven, not of earth, an integral part of man's spiritual equipment. But let me say, varying a little what has been said already, that all free fluent activity is abstract or separate in exactly the sense meant. Finding a use of language in all fluent activity is not at all opposed to the orthodox ideas of language. Forsooth, are writing and speaking the only cases of self-activity? Every spontaneous act, every expression of the living self shows, in the first place, an experience organized into a symbol or a past brought into adjustment with a present, and in the second place this symbol as something belonging to the active self, something which mediates the activity, something quite as much motive as stimulus, applied as a 'name,' or a 'report,' if you like, to an outer world. All action, I would assert, from the lowest to the highest, from the simplest to the most complex, is of a self naming a not-self. When action is, the object or the symbol is as much the agent as the subject. So, in the sense of language being original, one cannot object to thinking of it even as a gift from heaven; it is as original as activity. Biologically, freedom in an environmont is also freedom of an environment, and organism and environment are one as thinking-self and language are one.

It has not infrequently been a matter of controversy if thought were possible without language, and the solution of the problem seems to be that thought is possible without language

1 On the more general use of the term language compare the short discussion : 'A Psychological Interpretion of Certain Doctrines in Formal Logic.' PsYch. REv., Vol. III., No. 4, July, 18g6-pp. 422-426. 
in the narrower sense, that is, written and spoken language, but impossible without language as such, that is, without environment. Do animals think? Do they know relations? Are they addicted to language? Assuredly they are and do, if ever they act in self-expression. What living creature is not ' addicted' to an environment?

The rise of language as such, so our psychology here would lead us to conclude, means nothing more nor less than the mechanicalization of environment or the perceived world. In other words, the pure symbol, used in conception, in so far as a basis of fluent activity, is only a mechanism that the individual has become free to use. And in such mechanism, I would suggest in passing, as objective or physical, is evidence always of the rise of a community of individuals acting organically. In short, the fully developed object of perception is more than mere symbol; it is a mechanism in which is the basis of the life of a social organism. Its character as language, as 'medium of the exchange of thought,' can have no other import than this, since thought itself is organized social life. This intimate connection, moreover, between the rise of language and conception, the mechanicalization of environment, and the development of the social organism, is a most important outcome of the standpoint taken here, but discussion of it is not within the scope of the present article. Motive and stimulus are identical; environment is essentially linguistic; and language is not the medium of the exchange of abstract thought, but the basis of an organized life. That is the whole story in a nut-shell. Simply the linguistic environment makes possible individual self-expression in a social group; or mechanicism, like the a priori, which according to Kant makes the experience of it possible, is social. ${ }^{1}$

So far I have insisted on extending the use of the term language to make it include environment or the medium of expres-

'Social, I repeat, in the sense of free industrialism or of society as an organism; in history the social mechanicalism of Rome is evidently the ' a priori from ' in which modern life as industrial and organically international bas been possible. Rome, with her Christian idolatry, her spiritual monarchism, her linguistic formalism, her Jewish finance, only have witness to the originality of the medism of self-expression, an idea which the modern individual has naturally enough taken to himself. But, in general, mechanism is the o priori condition of individualism and organism. 
sion without limitation or abstraction. But, of course, language in the narrower sense, in the sense that limits it to special forms of sounds and shapes, has, even in its very narrowness, an important relation to the activity of thought. In emphasizing the broader view of language, therefore, I have appeared to slight the narrower. Hence I wish to add the following much condensed paragraphs by way of atonement.

It is a generally recognized principle that self-expression brings interpretation or meaning to the impulse expressed, and that meaning, coming so, controls the impulse. In other words, after expression impulse is held for a time, longer or shorter, in abeyance. Impulse in abeyance, however, not only confines the self's activity within the self, but also changes the special centres or organs of consciousness, and the confinement and the change would seem to be what make language in the narrower sense. Thus, to give the most obvious illustration, an impulse of man's in abeyance does not mean inactivity, but activity abstracting itself and identifying itself with eyes and ears, with the writer's hand and the speaker's tongue. Where, indeed, could activity find itself more at home than in these marvellously mobile organs? They are, in fact, but the stage upon which the self rehearses its part. 'They show the self acting ' to itself,' as we say specially of a child that learns to read without speaking; that is to say, acting apart or abstractly or reflectively.

Control, then, abstracts activity and develops very mobile organs for the special function so arising, the function of acting to oneself or quite within oneself. But acting to oneself brings the consciousness of environment or not-self; and, more than this, the environment gets what, in lack of a better account, I have to call a double character. Thus there arises a special consciousness, or experience, inhering in the special organs of the abstract activity and, at the same time, a special consciousness inhering in the organs of the self's complete activity, and, obviously enough, the special object of the former serves as a name or symbol of the latter; the special object of the organs of activity to oneself names the special object of the organs of the possible activity to one's world. The self does not talk talks nor see sights, nor hear sounds; it names the not-self. 
And, furthermore, in the name, arising as the object or natural medium of the abstracted activity, relationship, that is, relational or organic structure, will far outweigh all consideration of mere size. The name, in short, will be only a sort of after-image of the sphere of the self's original expression of impulse-original, that is, antecedent to the rise of control. That the original expression will have already determined the relations, or given the self an experience of them, is clear enough, since without such determination the expression itself could never have taken place. So the reproduction in an after-image is no miracle, but only shows how realized relationship in experience brings independence of mere quantitative determinations. The theory of language as originating in pictures, reduced reproductions of natural objects, has its limitations, but it will serve here in illustration. Its limitations, after all, are rather in terms of narrow application than of principle involved.

So, in summary, expression of impulse puts impulse in abeyance; impulse in abeyance brings an after-image of the special experience, which, as a relational whole, expression has defined; and, the after-image being a freed image, or the sphere of an abstracted activity, the direct use of it, the use of it with reference to its origin, the controlled, mediated use of it, gives what we commonly understand as the linguistic expression of self.

But we have yet to consider the last stage of knowledge, intuition. Intuition, however, is but the perfect freedom of using language, or of adaptation to environment. It is a stage of knowledge very much as sensation was a stage of knowledge. Thus the used mechanism is, as it were, the limit that the sensuously qualified symbol approaches, and intuition as stage of knowledge is a limit too, being such a limit as we have seen sensation to be and giving evidence of the same law of knowledge. Intuition comes with the completion of the process of mediation; with it consciousness ripens into fluent action; with it thought is set free. If in sensation stimulus and motive are one, in intuition at the other end of the scale developed mechanism as the stimulus and free agent as the motive, in short, language and thought, are one. The mechanism is nothing more nor less than stimulus to the free agent's will. 
So at both ends of the scale evolutional psychology has erred. It has retained intuition as the last stage of knowledge with the same blind persistence, or rather with the same misunderstanding of the true meaning, that has characterized its treatment of simple sensations or of sensation as stimulus or original continuum. Neither sensation nor intuition is a content of consciousness. The former is the vital impulse to selfexpression; the latter is that impulse fully mediated in an act. As stages of knowledge they are limits, the infinitesimal and the infinite respectively, and, so understood, they only show how psychology, bent on keeping knowledge in a sphere quite by itself, has striven to do without physiology and biology. Thus, again, sensation as stage of knowledge is the back-door by which psychology has spirited life into the domain of knowledge; and intuition in its turn is but an epistemological disguise for the ripened act; and if the former is due to the gratuitous construction of retrospection-on the part, say, of self-conscious inactivity-the latter results from a closely related prospection.

And the change in psychology, finally, that recognition of this origin of the evolutional stages of knowledge effects is simply the turning of the old-time idea, or concept, into an act; of self-conscious inactivity into activity; of psychology, science of the soul, into biology, the science of life on earth. 